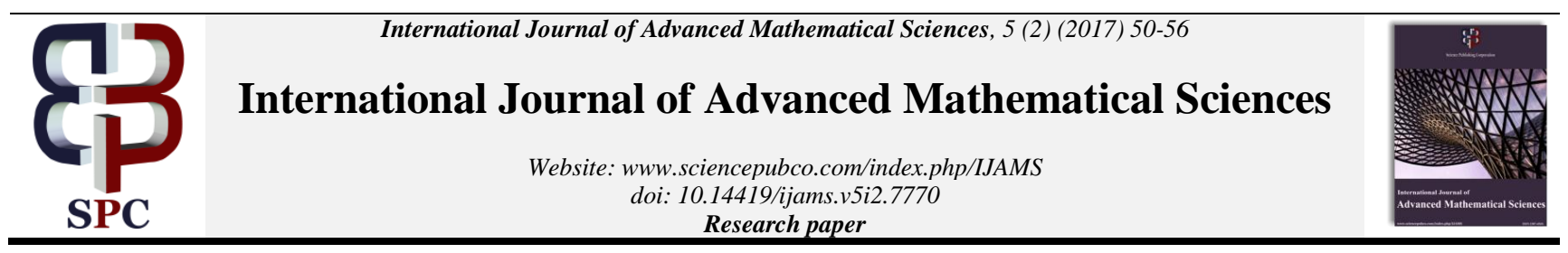

\title{
Globally exponential synchronization criterion of chaotic oscillators using active control
}

\author{
Israr Ahmad *, Sharifa Al Mahrouqi, Mohammad Shahzad \\ College of Applied Sciences Nizwa, Ministry of Higher Education, Oman \\ *Corresponding author E-mail: iak_2000plus@yahoo.com
}

\begin{abstract}
This research paper focuses the globally exponential synchronization between two identical and two nonidentical chaotic oscillators. With the help of Lyapunov direct method and using the active control technique, suitable algebraic conditions are obtained analytically that establish the globally exponential synchronization. The proposed globally exponential synchronization criterion is more general and much less conservative than the previously published works. A comparison, based upon the synchronization speed, cost and quality have been performed with our study of the previously published results. The effect of unknown external disturbances has also been discussed. Numerical simulation results are presented to illustrate the performance and efficiency of this study.
\end{abstract}

Keywords: Chaos Synchronization; Active Control; Lyapunov Stability Theory; Extended Bonhoeffer-Van Der Pol Oscillator; Chen Oscillator.

\section{Introduction}

After the remarkable work in [1], chaotic synchronization has been widely investigated due to its theoretical challenges and potential applications in different scientific fields [2-4]. A variety of chaos synchronization control techniques have been developed. These include nonlinear control [5], active control [6], adaptive control [7], lag and anticipated synchronizations [8], projective synchronization [9], linear feedback control [10], and so on. Among the aforesaid control techniques, the active control strategy for chaos synchronization has attracted a huge interest in the relevant literature because of its application to many practical systems [11-15].

Till to date, different active control strategies have been developed to address the full order synchronization [11-15]. Most of these active control schemes establish the globally exponential synchronization (GES) by allocating the eigenvalues of the matrix associated with the linear part of the closed-loop system to the left half of the complex plane. A large control effort is required to accomplish the GES. These types of control schemes are known for computing large control signals [16]. In addition, most of these active synchronization control methods are not robust with respect to the unknown external disturbances. Furthermore, the determination of exponential convergence rate has rarely been determined.

From control system theory [17], it is well-known that the exponential stability is the strongest from of stability, since, it provides a speedier convergence rate to the equilibrium point and a faster way to transfer the information signals from the transmitter to the receiver [18]. Thus, studies on the GES is a challenging topic and is seldom found in the literature. Therefore, it is significant as well as important to investigate the GES problem of chaotic systems, which may lead to develop a fore advanced theory and method in the study of secure communications. The present work is the improvement of the past published results [15], [18].

In this paper, the corresponding framework of synchronization will be set up to achieve the GES between two identical extended Bonhoeffer Van-der Pol (BVP) oscillators [15] and two nonidentical
BVP and Chen chaotic [19] oscillators using the active control technique. The globally exponential stability of the closed-loop is shown using the direct Lyapunov method [16]. The proposed GES criterion is much less conservative and more general as compared to the previously published works [15], [18]. Furthermore, in terms of synchronization quality, speed, robustness and cost of the controller design, a comparative study has been performed between the present results in this paper with previous published works [15], [18] to validate the performance of the current approach.

The rest of the paper is organized as follows. In Section 2, a theory for the proposed GES using active control technique is given. Section 3 presents the description of the BVP and Chen chaotic oscillators. The GES between two identical BVP chaotic oscillators is discussed in Section 4, followed by the GES between two nonidentical BVP and Chen chaotic oscillators in Section 5. Finally, the conclusion is drawn in Section 6, with some suggested future works.

\section{A theory for the globally exponential syn- chronization criterion}

\subsection{Problem statement}

Let us consider an autonomous chaotic system described as follows:

$$
\dot{\boldsymbol{x}}(t)=A \boldsymbol{x}(t)+f(\boldsymbol{x}(t))
$$

where $\dot{\boldsymbol{x}}(t)=\left[x_{1}(t)+x_{2}(t)+\cdots+x_{n}(t)\right]^{T} \in R^{n}$ is the state vector, is the state vector, $f \in R^{n}$ is the nonlinear continuous vector function and $A \in R^{n \times n}$ is the scaling function matrix.

Consider a master-slave system synchronization for two coupled chaotic systems (1) is given by:

(Master system)

$\dot{\boldsymbol{x}}(t)=A_{1} \boldsymbol{x}(t)+f_{1}(\boldsymbol{x}(t))+D(t)$

(Slave system)

$\dot{\boldsymbol{y}}(t)=A_{2} \boldsymbol{y}(t)+f_{2}(\boldsymbol{y}(t))+d(t)+\boldsymbol{u}(t)$

where $\dot{\boldsymbol{x}}(t)=\left[x_{1}(t)+x_{2}(t)+\cdots+x_{n}(t)\right]^{T} \in R^{n}$ and 
$\dot{\boldsymbol{y}}(t)=\left[y_{1}(t)+y_{2}(t)+\cdots+y_{n}(t)\right]^{T} \in R^{n}$ are the states vectors, $f_{1} \in R^{n}$ and $f_{2} \in R^{n}$ are the nonlinear continuous vector functions without parameters and $A_{1}, A_{2} \in R^{n}$ are the scaling function matrices of the master and slave systems (2), respectively. $D(t)$ and $d(t)$ are the unknown external disturbances present in the master and slave systems (2), respectively. The control input is represented by $\boldsymbol{u}(t) \in R^{n}$.

Definition 2.1: The synchronization error system is defined as the difference between the master and slave systems (2) that is described as follows:

$$
\|\boldsymbol{e}(t)\|_{2}=\|\boldsymbol{y}(t)-\boldsymbol{x}(t)\|_{2},
$$

where $\|$.$\| represents the Euclidean norm.$

Thus, the error system for the master-slave system synchronization (2) is described as follows:

$$
\begin{aligned}
\dot{\boldsymbol{e}}(t)= & A_{2} \boldsymbol{y}(t)-A_{1} \boldsymbol{x}(t)+f_{2}(\boldsymbol{y}(t))-f_{1}(\boldsymbol{x}(t))+D(t)- \\
& d(t)+\boldsymbol{u}(t), \\
= & A_{3} \boldsymbol{e}(t)+h(\boldsymbol{x}(t), \boldsymbol{y}(t))+D(t)-d(t)+\boldsymbol{u}(t),
\end{aligned}
$$

where $h(\boldsymbol{x}(t), \boldsymbol{y}(t))$ is the function that contains the nonlinear terms and un-common parts and $A_{3}=A_{2}-A_{1}$.

If $A_{1}=A_{2}$ and/or $f_{1}=f_{2}$, then, $\boldsymbol{x}(t)$ and $\boldsymbol{y}(t)$ are the states of two identical chaotic systems, and if $A_{1} \neq A_{2}$ and $f_{1} \neq f_{2}$, then, $\boldsymbol{x}(t)$ and $\boldsymbol{y}(t)$ are the states of two different chaotic systems.

Assumption 2.1: It is assumed that the unknown external disturbances $D(t)$ and $d(t)$ are the norm-bounded in $C^{1}$ [20]. i.e.

$$
|D(t)| \leq E^{m} \text { and }|d(t)| \leq E^{s} \text {. }
$$

Thus, from (4), it can be concluded that:

$$
|d(t)-D(t)| \leq \Delta
$$

where $\Delta=E^{m}+E^{s}$ and $E^{m}, E^{s}$ and $\Delta$ are unknown positive constants.

\subsection{Controller design}

The main goal of this subsection is to design such an active contro strategy that the limit of the error vector; $\boldsymbol{e}(t)=\left[e_{1}(t), e_{2}(t), \ldots+\right.$ $\left.e_{n}(t)\right]^{T}$ tends to zero globally exponentially. i.e.

$$
\lim _{t \rightarrow \infty}\|\boldsymbol{e}(t)\|_{2}=\lim _{t \rightarrow \infty}\|\boldsymbol{y}(t)-\boldsymbol{x}(t)\|_{2}=0 .
$$

Theorem 2.1: If the controller $\boldsymbol{u}(t)$ in (2) is designed such that the following inequality holds true:

$$
V(\boldsymbol{e}(t)) \leq V(0) \exp \left(-\frac{q_{(\min )}(Q)}{p_{(\max )}(P)} t\right),
$$

where $q_{(\min )}$ and $p_{(\max )}$, represent the minimum and maximum eigenvalues of two positive definite matrices (PDM) $Q$ and $P$, respectively then, the two coupled chaotic systems (2) are globally exponentially synchronized.

Proof of Theorem 2.1: Let us define the following active control function as:

$$
\boldsymbol{u}(t)=-h(\boldsymbol{x}(t), \boldsymbol{y}(t))-\Delta-K \boldsymbol{e}(t),
$$

where $K=\operatorname{diag}\left[k_{1}, k_{2}, \ldots, k_{n}\right]$ is a linear controller gain matrix which will be constructed. Using system of Eqs. (3) and (7) that yields:

$$
\begin{aligned}
\dot{\boldsymbol{e}}(t) & =A_{3} \boldsymbol{e}(t)-K \boldsymbol{e}(t) \\
& =\left(A_{3}-K\right) \boldsymbol{e}(t) \\
& =-\left(K-A_{3}\right) \boldsymbol{e}(t) .
\end{aligned}
$$

Eq. (8) describes the closed-loop system and the synchronization problem can be addressed as the stabilization of the closed-loop system at the origin. Let us construct the following Lyapunov error function as:

$$
V(\boldsymbol{e}(t))=\boldsymbol{e}^{T}(t) P \boldsymbol{e}(t) \geq 0, i=1,2, \ldots, n,
$$

where $V(\boldsymbol{e}(t)): R^{n} \rightarrow R^{n}$ is a positive definite function with $P=\operatorname{diag}\left[p_{1}, p_{2}, \ldots, p_{n}\right]$; is a positive definite matrix (PDM). Further, it is assumed that all the parameters and variables of the two coupled chaotic systems (2) are available and measureable. Noted that the closed-loop system in Eq. (8) is a linear system. The use of Lyapunov function to such a system is to establish the GES stability and an idea of tuning the controller parameters for the suitable position in the left half of the complex plane.

Let $p_{(\max )}$ and $p_{(\min )}$, respectively represent the minimum and maximum eigenvalues of the PDM $P$, then, from Eq. (9), it can be easily check that [21];

$$
\begin{array}{cc}
\quad p_{(\min )}(P) \sum_{1}^{n} e_{i}^{2}(t) \leq V(\boldsymbol{e}(t)) \leq p_{(\max )}(P) \sum_{1}^{n} e_{i}^{2}(t) \\
& \frac{V(\boldsymbol{e}(t))}{p_{(\max )}(P)} \leq \sum_{1}^{n}\left|e_{i}(t)\right|^{2} .
\end{array}
$$

The time derivative of the Lyapunov error function (9) along (8) is given as follows:

$$
\dot{V}(\boldsymbol{e}(t))=-2 P \sum_{1}^{n}\left(k_{i}-a_{i i}\right) e_{i}^{2}(t),
$$

where $a_{i i}(i=1,2, \ldots, n)$ are the coefficients of the matrix $A_{3}$. Rewrite Eq. (11) as follows:

$\dot{V}(\boldsymbol{e}(t))=$

$$
\begin{aligned}
& -\boldsymbol{e}^{T}(t)\left[\begin{array}{ccccc}
2 p\left(k_{1}-a_{11}\right) & 0 & & \cdots & 0 \\
\vdots & 2 p\left(k_{1}-a_{11}\right) & & \cdots & 0 \\
0 & \vdots & \ddots & & \vdots \\
0 & 0 & \cdots & 2 p\left(k_{1}-a_{11}\right)
\end{array}\right] \boldsymbol{e}(t) \\
& \Rightarrow 0 \\
& \quad \dot{V}(\boldsymbol{e}(t))=-\boldsymbol{e}^{T}(t) \boldsymbol{Q} \boldsymbol{e}(t)
\end{aligned}
$$

where

$$
Q=\left[\begin{array}{ccccc}
2 p\left(k_{1}-a_{11}\right) & 0 & & \cdots & 0 \\
\vdots & 2 p\left(k_{1}-a_{11}\right) & & \cdots & 0 \\
0 & \vdots & \ddots & & \vdots \\
0 & 0 & \cdots & 2 p\left(k_{1}-a_{11}\right)
\end{array}\right]
$$

is a PDM. Now from Eq. (12), it follows that:

$$
\begin{array}{cc} 
& \dot{V}(\boldsymbol{e}(t))=-Q \sum_{1}^{n} e_{i}^{2}(t) \\
\Rightarrow & \quad \dot{V}(\boldsymbol{e}(t)) \leq-q_{(\min )}(Q) \sum_{1}^{n}\left|e_{i}(t)\right|^{2}
\end{array}
$$

Using Eqs (10) and (14) that yields:

$$
\dot{V}(\boldsymbol{e}(t))=-q_{(\min )}(Q) \frac{V(\boldsymbol{e}(t))}{p_{(\max )}(P)} .
$$

Integrating the inequality (15) from $0 \rightarrow t$, results in the following Lyapunov estimation:

$$
V(\boldsymbol{e}(t)) \leq V(0) \exp \left(-\frac{q_{(\min )}(Q)}{p_{(\max )}(P)} t\right) .
$$

The inequality (16) accomplishes the globally exponential stability of the closed-loop (8) at the origin. Thus, the two coupled chaotic systems (2) are globally exponentially synchronized.

This completes the proof.

Remark 2.1: The term $\left(\frac{q_{(\min )}(Q)}{p_{(\max )}(P)}\right)$ in Eq. (16) determines the exponential convergence rate of the synchronized error system to the origin.

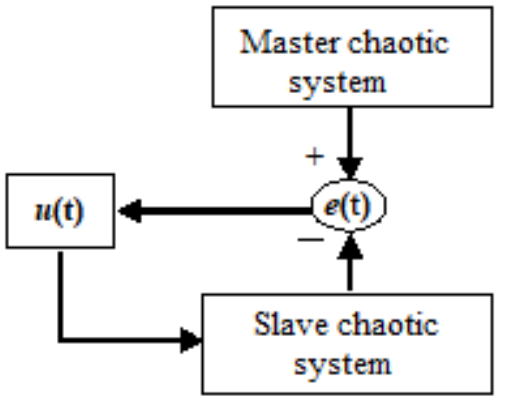

Fig. 1: Master-Slave system synchronization scheme.

\section{Description of the chaotic systems}

\subsection{The extended Bonhoeffer van-der pol chaotic oscilla- tor}

Electric circuits are one of the natural physical systems and it can be characterized by a variety of equations [22]. The multi-scroll chaotic attractors exhibited by electric circuits have received considerable attention of the researchers theoretically as well as experimentally [23]. The three-dimensional BVP [23] is one of the elec- 
tric circuits, which realizes chaos and shows very rich, complex dynamic behavior and yet has potential applications. An extended BVP oscillator can show a stable oscillator as well as a stable threshold phenomenon [23]. The extended BVP oscillator has been observed numerically and experimentally in certain systems and has attributes of great utility to medicine, chemical kinetics, neuroscience, electrical circuits and secure communications [15], [23-25]. The extended BVP model consists of an inductor, two capacitors and a linear resistor. By applying Kirchoff's laws, the circuitry equation of the extended BVP oscillator [15] is described as follows:

$$
C \frac{d v_{1}}{d t}=-i-g\left(v_{1}\right), C \frac{d v_{2}}{d t}=-i-\frac{v_{2}}{r}, L \frac{d i}{d t}=v_{1}-v_{2} \text {, }
$$

where $g\left(v_{1}\right)=-a v_{1}-b \tanh \left(c v_{1}\right)$ indicates the $v-i$ characteristics of the nonlinear resistor [15].

Using the following identities:

$A=a \sqrt{\frac{L}{C}}, B=\frac{1}{r} \sqrt{\frac{L}{C}}, \delta=b c \sqrt{\frac{1}{L C}}, r=\sqrt{\frac{1}{L C}} t, x=\frac{v_{1}}{b} \sqrt{\frac{C}{L}}, y=\frac{v_{2}}{b} \sqrt{\frac{C}{L}}$.

Thus, the normalized dynamical model of an extended BVP oscillator (17) can be described as follows:

$$
\left[\begin{array}{l}
\dot{x}(t) \\
\dot{y}(t) \\
\dot{z}(t)
\end{array}\right]=\left[\begin{array}{ccc}
A & 0 & -1 \\
0 & -B & 1 \\
1 & -1 & 0
\end{array}\right]\left[\begin{array}{l}
x(t) \\
y(t) \\
z(t)
\end{array}\right]+\left[\begin{array}{c}
\tanh \delta x(t) \\
0 \\
0
\end{array}\right],
$$

where $[x(t), y(t), z(t)] \in R^{3}$ are the state variables correspond to $v_{1}, v_{2}$ and $i$ respectively and $A, B$ and $\delta$ are the parameters of the BVP (18) with $\delta$ corresponds to the value of a fixed resistor $r$. The extended BVP oscillator exhibits a chaotic attractor with initial condition $(-0.5,-1,0.5)$ and parameters values $A=1, B=1.2$ and $\delta=1$ as shown in Figure 2(A).

\subsection{The Chen chaotic oscillator}

The vector form of the chaotic Chen oscillator [19] is described as follows:

$$
\left[\begin{array}{l}
\dot{x}(t) \\
\dot{y}(t) \\
\dot{z}(t)
\end{array}\right]=\left[\begin{array}{ccc}
-\alpha & \alpha & 0 \\
-v & \gamma & 0 \\
0 & 0 & -\beta
\end{array}\right]\left[\begin{array}{c}
x(t) \\
y(t) \\
z(t)
\end{array}\right]+\left[\begin{array}{c}
0 \\
-x(t) z(t) \\
x(t) y(t)
\end{array}\right],
$$

where $[x(t), y(t), z(t)] \in R^{3}$ are the corresponding state vectors and $\alpha, \beta, \gamma$ and $v$ are the positive parameters of the Chen chaotic system. The Chen system (19) exhibits chaos for the parameters values $\alpha=35, \beta=3, \gamma=28$ and $v=7$, as shown in Figure 2(B).

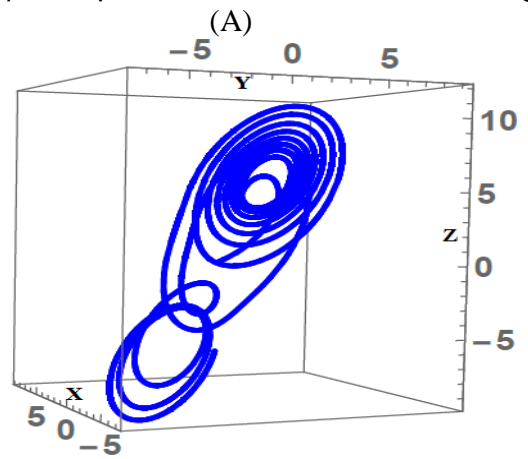

(B)

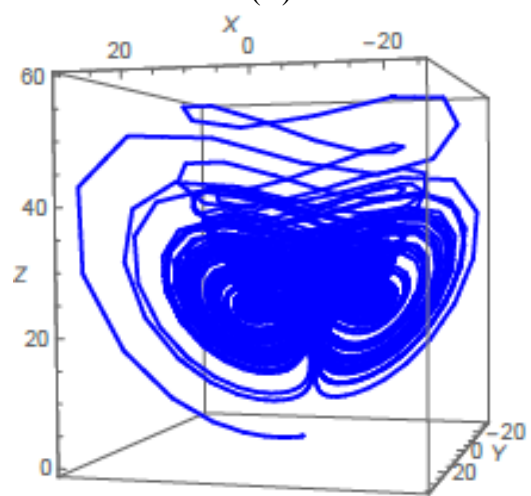

Fig. 2: 3-D Phase portrait of the chaotic (A) BVP oscillator, (B) Chen oscillator.

\section{Synchronization between two identical ex- tended Bonhoeffer-van der pol oscillators}

\subsection{Problem statement}

In order to observe the globally exponential complete synchronization behavior of an extended BVP oscillator (18), we used two nearly identical BVP oscillators, where the master BVP oscillator is denoted by the subscript 1 and the slave BVP oscillator having identical equations denoted by the subscript 2 . The complete synchronization is then described as follows: (Master oscillator)

$$
\left[\begin{array}{l}
\dot{x}_{1}(t) \\
\dot{y}_{1}(t) \\
\dot{z}_{1}(t)
\end{array}\right]=\left[\begin{array}{ccc}
A & 0 & -1 \\
0 & -B & 1 \\
1 & -1 & 0
\end{array}\right]\left[\begin{array}{l}
x_{1}(t) \\
y_{1}(t) \\
z_{1}(t)
\end{array}\right]+\left[\begin{array}{c}
\tanh \delta x_{1}(t) \\
0 \\
0
\end{array}\right]+\left[\begin{array}{c}
D_{1}(t) \\
D_{2}(t) \\
D_{3}(t)
\end{array}\right]
$$
(Slave oscillator)

$$
\begin{aligned}
{\left[\begin{array}{l}
\dot{x}_{2}(t) \\
\dot{y}_{2}(t) \\
\dot{z}_{2}(t)
\end{array}\right]=} & {\left[\begin{array}{ccc}
A & 0 & -1 \\
0 & -B & 1 \\
1 & -1 & 0
\end{array}\right]\left[\begin{array}{l}
x_{2}(t) \\
y_{2}(t) \\
z_{2}(t)
\end{array}\right]+\left[\begin{array}{c}
\tanh \delta x_{2}(t) \\
0 \\
0
\end{array}\right]+\left[\begin{array}{l}
d_{1}(t) \\
d_{2}(t) \\
d_{3}(t)
\end{array}\right]+} \\
& {\left[\begin{array}{l}
u_{1}(t) \\
u_{2}(t) \\
u_{3}(t)
\end{array}\right] }
\end{aligned}
$$

where

$\left[x_{1}(t), y_{1}(t), z_{1}(t)\right]^{T} \in R^{3}$ and $\left[x_{2}(t), y_{2}(t), z_{2}(t)\right]^{T} \in R^{3}$ are the corresponding state vectors and $A, B$ and $\delta$ are the positive parameters of the master and slave oscillators (19), respectively. $D_{i}(t)$ and $d_{i}(t)$ for $i=1,3$ are the unknown external disturbances present in the master and slave oscillators (19), respectively where $\left[u_{1}(t), 0, u_{3}(t)\right]^{T} \in R^{3}$ is the control input vector. The controller $\boldsymbol{u}(t)=\left[u_{i}(t)\right]^{T}$ is to be designed to solve the synchronization problem for two identical BVP oscillators (20) with identical parameters $A, B$ and $\delta$ in spite of the dissimilarities in their initial conditions.

For the complete synchronization (20), the synchronization error systems is described as follows:

$$
\begin{aligned}
& {\left[\begin{array}{l}
\dot{e}_{1}(t) \\
\dot{e}_{2}(t) \\
\dot{e}_{3}(t)
\end{array}\right]=\left[\begin{array}{ccc}
A & 0 & -1 \\
0 & -B & 1 \\
1 & -1 & 0
\end{array}\right]\left[\begin{array}{l}
e_{1}(t) \\
e_{2}(t) \\
e_{3}(t)
\end{array}\right]+\left[\begin{array}{l}
d_{1}(t)-D_{1}(t) \\
d_{2}(t)-D_{2}(t) \\
d_{3}(t)-D_{3}(t)
\end{array}\right]+} \\
& {\left[\begin{array}{c}
\tanh \delta x_{2}(t)-\tanh \delta x_{1}(t) \\
0 \\
0
\end{array}\right]+\left[\begin{array}{l}
u_{1}(t) \\
u_{2}(t) \\
u_{3}(t)
\end{array}\right],}
\end{aligned}
$$

where $\left\|e_{i}(t)\right\|_{2}=\left\|y_{i}(t)-x_{i}(t)\right\|_{2}, \quad i=1,2,3$.

The controlled slave BVP oscillator is synchronous with the master BVP oscillator iff:

$$
\lim _{t \rightarrow \infty}\left\|e_{i}(t)\right\|_{2}=\lim _{t \rightarrow \infty}\left\|y_{i}(t)-x_{i}(t)\right\|_{2}=0, i=1,2,3 .
$$

\subsection{Controller design}

Theorem 4.1: If the controller $\boldsymbol{u}(t)=\left[u_{i}(t)\right]^{T}$ in (20) is designed such that the following inequality holds true:

$$
V(\boldsymbol{e}(t)) \leq V(0) \exp \left(-2 \frac{q_{(\min )}(Q)}{p_{(\max )}(P)} t\right),
$$

where $q_{(\min )}$ and $p_{(\max )}$, represent the minimum and maximum eigenvalues of two positive definite matrices (PDM) $Q$ and $P$, respectively then, the two identical BVP chaotic oscillators (20) are globally exponentially synchronized.

Proof of Theorem 4.1. Let us construct the active control functions as follows:

$$
\begin{gathered}
{\left[\begin{array}{l}
u_{1}(t) \\
u_{2}(t) \\
u_{3}(t)
\end{array}\right]=} \\
{\left[\begin{array}{ccc}
k_{1} & 0 & 0 \\
0 & 0 & 0 \\
0 & 0 & k_{3}
\end{array}\right]\left[\begin{array}{l}
e_{1}(t) \\
e_{2}(t) \\
e_{3}(t)
\end{array}\right]-\left[\begin{array}{l}
\Delta_{1} \\
\Delta_{2} \\
\Delta_{3}
\end{array}\right]-} \\
0 \\
0
\end{gathered}
$$


where $k_{1}$ and $k_{3}$ are the controller gain coefficients, which will be computed. Using systems of Eqs. (21) and (23), the closed-loop system is given as follows:

$$
\left[\begin{array}{l}
\dot{e}_{1}(t) \\
\dot{e}_{2}(t) \\
\dot{e}_{3}(t)
\end{array}\right]=\left[\begin{array}{ccc}
A-k_{1} & 0 & -1 \\
0 & -B & 1 \\
1 & -1 & -k_{3}
\end{array}\right]\left[\begin{array}{l}
e_{1}(t) \\
e_{2}(t) \\
e_{3}(t)
\end{array}\right]
$$

In order to establish the globally exponential stability and convergence of the closed-loop system (24) at the origin, let us construct the following Lyapunov error function as:

$$
V(\boldsymbol{e}(t))=\boldsymbol{e}^{T}(t) P \boldsymbol{e}(t) \geq 0,
$$

where

$$
P=\operatorname{diag}[0.5,0.5,0.5],
$$

and $V(\boldsymbol{e}(t)): R^{3} \rightarrow R^{3}$ is a positive definite. Now, the time derivative of the Lyapunov function (26) is given as follows:

$$
\dot{V}(\boldsymbol{e}(t))=\left[e_{1}(t) \dot{e}_{1}(t)+e_{2}(t) \dot{e}_{2}(t)+e_{3}(t) \dot{e}_{3}(t)\right]
$$

Using system of Eqs (24) and (27) that yields:

$$
\begin{aligned}
\dot{V}(\boldsymbol{e}(t))=-\left[\begin{array}{c}
e_{1}(t) \\
e_{2}(t) \\
e_{3}(t)
\end{array}\right]^{T}\left[\begin{array}{ccc}
k_{1}-A & 0 & 0 \\
0 & B & 0 \\
0 & 0 & k_{3}
\end{array}\right]\left[\begin{array}{l}
e_{1}(t) \\
e_{2}(t) \\
e_{3}(t)
\end{array}\right] \\
\dot{V}(\boldsymbol{e}(t))=-\boldsymbol{e}^{T}(t) Q \boldsymbol{e}(t),
\end{aligned}
$$

where

$$
Q=\left[\begin{array}{ccc}
k_{1}-A & 0 & 0 \\
0 & B & 0 \\
0 & 0 & k_{3}
\end{array}\right]
$$

Since $A>0$ and $B>0$, thus, if the linear controller feedback gains $k_{1}$ and $k_{3}$ satisfy the following conditions:

$$
k_{1}>0 \text { and } k_{3}>0 \text {, }
$$

then, the matrix $Q$ is PDM and $\dot{V}(\boldsymbol{e}(t))$ is a negative definite function. From Eq. (26), we have:

$$
\begin{aligned}
V(\boldsymbol{e}(t))= & {\left[\begin{array}{ccc}
p_{1} & 0 & 0 \\
0 & p_{2} & 0 \\
0 & 0 & p_{3}
\end{array}\right]\left[e_{1}^{2}(t)+e_{2}^{2}(t)+e_{3}^{2}(t)\right] } \\
= & {\left[\begin{array}{ccc}
0.5 & 0 & 0 \\
0 & 0.5 & 0 \\
0 & 0 & 0.5
\end{array}\right]\left[e_{1}^{2}(t)+e_{2}^{2}(t)+e_{3}^{2}(t)\right] } \\
= & 0.5\left[\begin{array}{ccc}
1 & 0 & 0 \\
0 & 1 & 0 \\
0 & 0 & 1
\end{array}\right]\left[e_{1}^{2}(t)+e_{2}^{2}(t)+e_{3}^{2}(t)\right] \\
\Rightarrow \quad & V(\boldsymbol{e}(t))=\left[e_{1}^{2}(t)+e_{2}^{2}(t)+e_{3}^{2}(t)\right]
\end{aligned}
$$

Let $p_{(\max )}$ and $p_{(\min )}$, respectively represents the minimum and maximum eigenvalue of the PDM $P$, then, from Eq. (25), it follows that:

$$
\begin{gathered}
\frac{p_{(\min )}(P)}{2} \sum_{1}^{3} e_{i}^{2}(t) \leq V(\boldsymbol{e}(t)) \leq \frac{p_{(\max )}(P)}{2} \sum_{1}^{3} e_{i}^{2}(t) \\
2 V(\boldsymbol{e}(t)) \leq p_{(\max )}(P) \sum_{1}^{3} e_{i}^{2}(t)
\end{gathered}
$$

From Eq. (27), let us suppose that $q_{(\min )} Q=\min \left\{k_{1}-A, B, k_{3}\right\}$, then, Eq. (27) implies that:

$$
\begin{aligned}
\dot{V}(\boldsymbol{e}(t)) & \leq-q_{(\min )}(Q)\left[\begin{array}{l}
e_{1}(t) \\
e_{2}(t) \\
e_{3}(t)
\end{array}\right]^{T}\left[\begin{array}{l}
e_{1}(t) \\
e_{2}(t) \\
e_{3}(t)
\end{array}\right] \\
& \leq-q_{(\min )}(Q)\left[e_{1}^{2}(t)+e_{2}^{2}(t)+e_{3}^{2}(t)\right] \\
& \leq-q_{(\min )}(Q) \sum_{1}^{3}\left|e_{i}(t)\right|^{2} .
\end{aligned}
$$

Using inequalities (32) and (33) that yields:

$$
\dot{V}(\boldsymbol{e}(t)) \leq-2 \frac{q_{(\min )}(Q)}{p_{(\max )}(P)} V(\boldsymbol{e}(t)) .
$$

Integrating the inequality (34) from $0 \rightarrow t$, results in the following Lyapunov estimation:

$$
V(\boldsymbol{e}(t)) \leq V(0) \exp (-\Psi t),
$$

where

$$
\Psi=2 \frac{q_{(\min )}(Q)}{p_{(\max )}(P)} .
$$

Thus, the closed-loop system (24) is globally exponentially stable. Therefore, the two coupled chaotic BVP oscillators (20) are globally exponentially synchronized.

\subsection{Numerical simulation and discussion}

Numerical simulation results are given to verify the efficiency and performance of the proposed approach. The parameters for the BVP oscillator [15] are set as $A=1, B=1.2$ and $\delta=1$ with initial states are taken as $x_{1}(t)=-2, y_{1}(t)=2, z_{1}(t)=0$ and $x_{2}(t)=-0.5, y_{2}(t)=-1, z_{2}(t)=0.5$, respectively. the linear feedback gains of the controllers are selected as $k_{1}=2$ and $k_{3}=1$. In numerical simulation, the following unknown external disturbances are applied to the master and slave systems (20) respectively.

$$
\begin{aligned}
& D_{1}(t)=-0.2 \sin \left(\frac{3 \pi}{2} t\right), D_{2}(t)=0.1 \cos (\pi t), \\
& d_{1}(t)=0.15 \cos \left(\frac{\pi}{2} t\right), d_{2}(t)=-0.25 \sin \left(\frac{\pi}{3} t\right) .
\end{aligned}
$$

Accordingly, $\Delta_{1}=0.3$ and $\Delta_{2}=0.4$.

For the two identical BVP chaotic oscillators (20), the time histories of the convergence of the error states are displayed in Figure 3. It is observed that the complete GES is achieved within 4 seconds by using only two active control functions, while the complete synchronization objective in [15] is accomplished in $t \approx 10 \mathrm{~s}$ app., and three active control functions are utilized. These factors show the efficiency and performance of the proposed GES approach computationally. To quantify the synchronization, Figure 4 , illustrates the norm $\_2$ of the error vector $r(t)=\sqrt{e_{1}^{2}(t)+e_{2}^{2}(t)+e_{3}^{2}(t)}$ for the GES. The converging rate is determined by the constant $\Psi=2 \frac{q_{(\min )}(Q)}{p_{(\max )}(P)}$. The converging rate could be adjusted by the linear controller feedback gains and the matrix $P$. By numerical calculations, we can easily verify that $p_{(\max )}(P)=0.5$ and $q_{(\min )}(Q)=1$. Thus, from the main Theorem 4.1 , it is concluded that the two identical BVP chaotic oscillators (20) are globally exponentially synchronized with an exponential convergence rate $\Psi=4$.

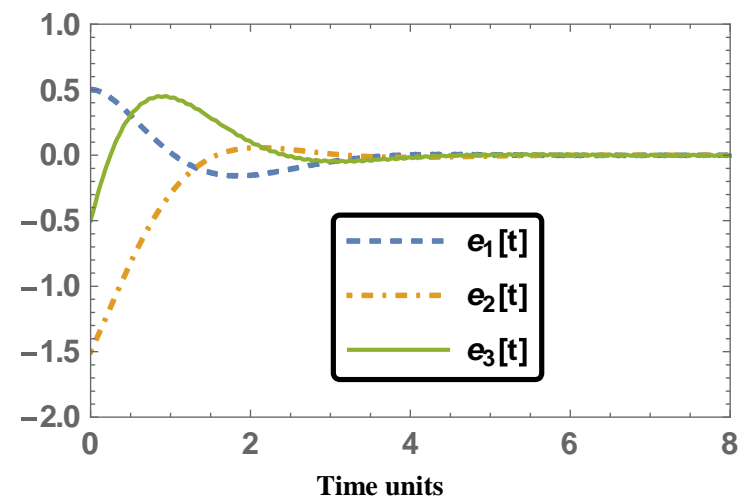

Fig. 3: Time Series of the Synchronized Error States.

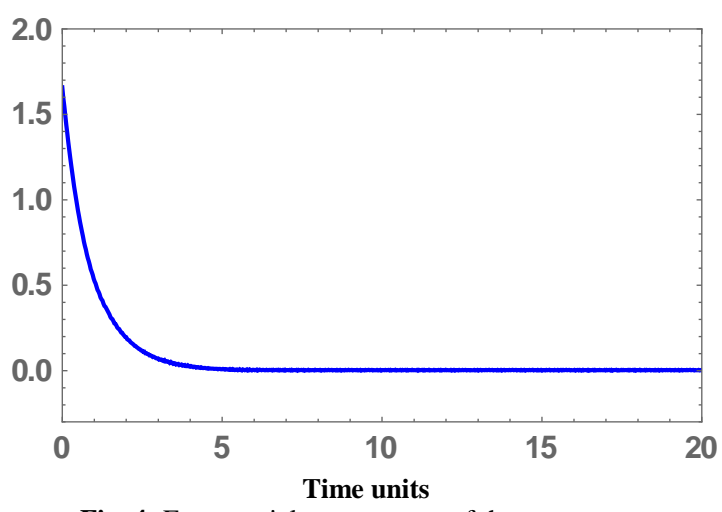

Fig. 4: Exponential convergence of the error states.

Figure 5, presents the time history of the control inputs (22). The proposed control strategy is economical and simple in implementation to accomplish the GES. 


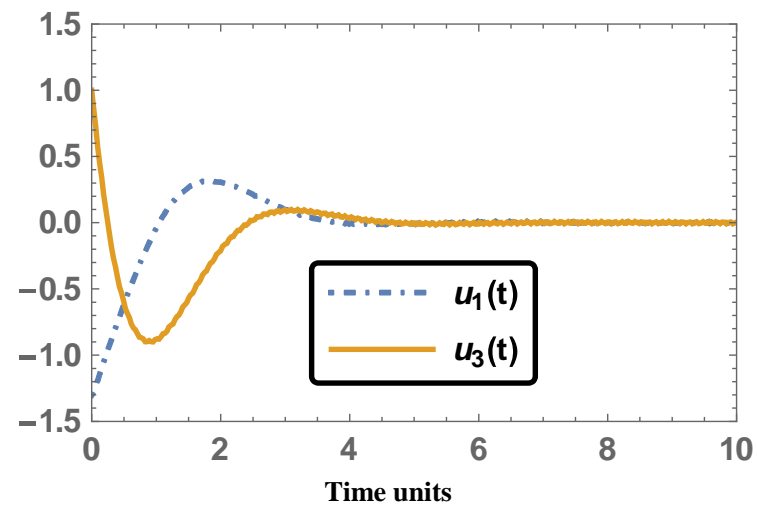

Fig. 5: Time Series of the control inputs $u_{1}(t)$ and $u_{3}(\mathrm{t})$

\section{Synchronization between two nonidentical extended Bonhoeffer-van der pol and Chen chaotic oscillators}

\subsection{Problem statement}

In this subsection of the paper, the proposed GES approach will be applied to achieve the synchronization between two nonidentical chaotic oscillators. The extended BVP oscillator is considered as the master system with the subscript 1 and the Chen chaotic oscillator is considered as the slave system with the subscript 2 . Therefore, the master-slave system synchronization is described as follows:

$$
\begin{aligned}
& \text { (Master oscillator) } \\
& {\left[\begin{array}{l}
\dot{x}_{1}(t) \\
\dot{y}_{1}(t) \\
\dot{z}_{1}(t)
\end{array}\right]=\left[\begin{array}{ccc}
A & 0 & -1 \\
0 & -B & 1 \\
1 & -1 & 0
\end{array}\right]\left[\begin{array}{l}
x_{1}(t) \\
y_{1}(t) \\
z_{1}(t)
\end{array}\right]+\left[\begin{array}{c}
\tanh \delta x_{1}(t) \\
0 \\
0
\end{array}\right]+\left[\begin{array}{c}
D_{1}(t) \\
D_{2}(t) \\
D_{3}(t)
\end{array}\right]}
\end{aligned}
$$
(Slave oscillator)

$$
\begin{aligned}
{\left[\begin{array}{l}
\dot{x}_{2}(t) \\
\dot{y}_{2}(t) \\
\dot{z}_{2}(t)
\end{array}\right]=} & {\left[\begin{array}{ccc}
-\alpha & \alpha & 0 \\
-v & \gamma & 0 \\
0 & 0 & -\beta
\end{array}\right]\left[\begin{array}{l}
x_{2}(t) \\
y_{2}(t) \\
z_{2}(t)
\end{array}\right]+\left[\begin{array}{c}
0 \\
-x_{2}(t) z_{2}(t) \\
x_{2}(t) y_{2}(t)
\end{array}\right]+\left[\begin{array}{l}
d_{1}(t) \\
d_{2}(t) \\
d_{3}(t)
\end{array}\right]+} \\
& {\left[\begin{array}{l}
u_{1}(t) \\
u_{2}(t) \\
u_{3}(t)
\end{array}\right], }
\end{aligned}
$$

where $\left[x_{1}(t), y_{1}(t), z_{1}(t)\right]^{T} \in R^{3}$ and $\left[x_{2}(t), y_{2}(t), z_{2}(t)\right]^{T} \in R^{3}$ are the corresponding states vectors of the master and slave oscillators (36), respectively. $A, B$ and $\delta$ are the positive parameters of the master system in Eq. (36) and $\alpha, \beta, \gamma$ and $v$ are the positive parameters of the slave system in Eq. (36). $D_{i}(t)$ and $d_{i}(t)$ for $i=1,2,3$ are the unknown external disturbances acting on the master and slave systems (36), respectively where $\left[u_{1}(t), u_{2}(t), u_{3}(t)\right]^{T} \in R^{3}$ represents the control input.

The error system for the nonidentical synchronization (36) is described as follows:

$$
\begin{aligned}
{\left[\begin{array}{l}
\dot{e}_{1}(t) \\
\dot{e}_{2}(t) \\
\dot{e}_{3}(t)
\end{array}\right]=} & {\left[\begin{array}{ccc}
-\alpha-A & \alpha & 1 \\
-v & \gamma+B & -1 \\
-1 & 1 & -\beta
\end{array}\right]\left[\begin{array}{l}
e_{1}(t) \\
e_{2}(t) \\
e_{3}(t)
\end{array}\right]+\left[\begin{array}{c}
-\tanh \delta x_{1}(t) \\
-x_{2}(t) y_{2}(t) \\
x_{2}(t) z_{2}(t)
\end{array}\right]+} \\
& {\left[\begin{array}{l}
d_{1}(t)-D_{1}(t) \\
d_{2}(t)-D_{2}(t) \\
d_{3}(t)-D_{3}(t)
\end{array}\right]+\left[\begin{array}{l}
u_{1}(t) \\
u_{2}(t) \\
u_{3}(t)
\end{array}\right] }
\end{aligned}
$$

where $\left\|e_{i}(t)\right\|_{2}=\left\|y_{i}(t)-x_{i}(t)\right\|_{2}, \quad i=1,2,3$.

The controlled slave Chen chaotic oscillator is synchronous with the master BVP chaotic oscillator iff:

$$
\lim _{t \rightarrow \infty}\left\|e_{i}(t)\right\|_{2}=\lim _{t \rightarrow \infty}\left\|y_{i}(t)-x_{i}(t)\right\|_{2}=0, i=1,2,3 .
$$

\subsection{Controller design}

Theorem 5.1: If the following in-equality holds true:

$$
V(\boldsymbol{e}(t)) \leq V(0) \exp \left(-2 \frac{q_{(\min )}(Q)}{p_{(\max )}(P)} t\right),
$$

where $q_{(\min )}$ and $p_{(\max )}$, represent the minimum and maximum eigenvalues of positive definite matrices (PDM) $Q$ and $P$, the two coupled chaotic oscillators (36) are globally exponentially synchronized.

Proof of Theorem 5.1. Let us construct the following active control functions as:

$$
\begin{aligned}
{\left[\begin{array}{l}
u_{1}(t) \\
u_{2}(t) \\
u_{3}(t)
\end{array}\right]=} & -\left(\left[\begin{array}{ccc}
-A & 0 & 1 \\
0 & B & -1 \\
-1 & 1 & 0
\end{array}\right]+\left[\begin{array}{ccc}
k_{1} & 0 & 0 \\
0 & k_{2} & 0 \\
0 & 0 & k_{3}
\end{array}\right]\right)\left[\begin{array}{l}
e_{1}(t) \\
e_{2}(t) \\
e_{3}(t)
\end{array}\right]+ \\
& {\left[\begin{array}{c}
\tanh \delta x_{1}(t) \\
x_{2}(t) z_{2}(t) \\
-x_{2}(t) y_{2}(t)
\end{array}\right]-\left[\begin{array}{l}
\Delta_{1} \\
\Delta_{2} \\
\Delta_{3}
\end{array}\right], }
\end{aligned}
$$

where $k_{1}, k_{2}$ and $k_{3}$ are the controller gain coefficients that adjust the strength of the feedback controller into the slave system. Using Eqs. (37) and (39), the closed-loop system (37) is given by:

$$
\left[\begin{array}{l}
\dot{e}_{1}(t) \\
\dot{e}_{2}(t) \\
\dot{e}_{3}(t)
\end{array}\right]=\left[\begin{array}{ccc}
-\left(\alpha+k_{1}\right) & \alpha & 0 \\
-v & \gamma-k_{2} & 0 \\
0 & 0 & -\left(\beta+k_{3}\right)
\end{array}\right]\left[\begin{array}{l}
e_{1}(t) \\
e_{2}(t) \\
e_{3}(t)
\end{array}\right] .
$$

Considering $k_{1}=k_{3}=0$ and rewrite system of Eq. (40) as follows:

$$
\left[\begin{array}{l}
\dot{e}_{1}(t) \\
\dot{e}_{2}(t) \\
\dot{e}_{3}(t)
\end{array}\right]=\left[\begin{array}{ccc}
-\alpha & \alpha & 0 \\
-v & \gamma-k_{2} & 0 \\
0 & 0 & -\beta
\end{array}\right]\left[\begin{array}{l}
e_{1}(t) \\
e_{2}(t) \\
e_{3}(t)
\end{array}\right] .
$$

Let us consider the Lyapunov function as follows:

$$
\begin{gathered}
V(\boldsymbol{e}(t))=\boldsymbol{e}^{T}(t) P \boldsymbol{e}(t) \geq 0, \\
V(\boldsymbol{e}(t))=P\left[e_{1}^{2}(t)+e_{2}^{2}(t)+e_{3}^{2}(t)\right],
\end{gathered}
$$

where $V(\boldsymbol{e}(t)): R^{3} \rightarrow R^{3}$ is a positive definite function with $P=\operatorname{diag}[0.5,2.5,0.5]$ is a PDM. Now, the time derivative of the Lyapunov function (43) is given as follows:

$$
\begin{aligned}
\dot{V}(\boldsymbol{e}(t)) & =P\left[2 e_{1}(t) \dot{e}_{1}(t)+2 e_{2}(t) \dot{e}_{2}(t)+2 e_{3}(t) \dot{e}_{3}(t)\right] \\
& =\left[e_{1}(t) \dot{e}_{1}(t)+5 e_{2}(t) \dot{e}_{2}(t)+e_{3}(t) \dot{e}_{3}(t)\right] .
\end{aligned}
$$

Using systems of Eqs (41) and (44), we have:

$$
\begin{aligned}
& \dot{V}(\boldsymbol{e}(t))=- {\left[\begin{array}{l}
e_{1}(t) \\
e_{2}(t) \\
e_{3}(t)
\end{array}\right]^{T}\left[\begin{array}{ccc}
\alpha & 0 & 0 \\
0 & 5\left(\gamma-k_{2}\right) & 0 \\
0 & 0 & \beta
\end{array}\right]\left[\begin{array}{l}
e_{1}(t) \\
e_{2}(t) \\
e_{3}(t)
\end{array}\right] } \\
& \dot{V}(\boldsymbol{e}(t))=-\boldsymbol{e}^{T}(t) Q \boldsymbol{e}(t),
\end{aligned}
$$

where

$$
Q=\left[\begin{array}{ccc}
\alpha & 0 & 0 \\
0 & 5\left(\gamma-k_{2}\right) & 0 \\
0 & 0 & \beta
\end{array}\right]
$$

Since $\alpha>0, \beta>0$ and $\gamma>0$, therefore, if $k_{2}<\gamma$, then, the matrix $Q$ becomes PDM and hence, $\dot{V}(\boldsymbol{e}(t))$ becomes a negative definite function. From Eq. (43), we have:

$$
\begin{aligned}
V(\boldsymbol{e}(t))= & {\left[\begin{array}{ccc}
0.5 & 0 & 0 \\
0 & 2.5 & 0 \\
0 & 0 & 0.5
\end{array}\right]\left[e_{1}^{2}(t)+e_{2}^{2}(t)+e_{3}^{2}(t)\right] } \\
= & 0.5\left[\begin{array}{lll}
1 & 0 & 0 \\
0 & 5 & 0 \\
0 & 0 & 1
\end{array}\right]\left[e_{1}^{2}(t)+e_{2}^{2}(t)+e_{3}^{2}(t)\right] \\
& 2 V(\boldsymbol{e}(t))=\left[e_{1}^{2}(t)+5 e_{2}^{2}(t)+e_{3}^{2}(t)\right] \\
& {\left[e_{1}^{2}(t)+e_{2}^{2}(t)+e_{3}^{2}(t)\right] \leq 2 V(\boldsymbol{e}(t)) . }
\end{aligned}
$$

Let $p_{(\max )}$ represents the maximum eigenvalue of the PDM $P$, then, from Eq. (43), it follows that:

$$
\frac{2 V(e(t))}{p_{(\max )}(P)} \leq \sum_{1}^{3} e_{i}^{2}(t)
$$

From Eq. (47), let us suppose that $q_{(\min )} Q=\min \{\alpha, 5(\gamma-$ $\left.k_{2}\right), \beta$, then, Eq. (46) implies that:

$$
\begin{aligned}
\dot{V}(\boldsymbol{e}(t)) & \leq-q_{(\text {min })}(Q)\left[\begin{array}{l}
e_{1}(t) \\
e_{2}(t) \\
e_{3}(t)
\end{array}\right]^{T}\left[\begin{array}{l}
e_{1}(t) \\
e_{2}(t) \\
e_{3}(t)
\end{array}\right] \\
& \leq-q_{(\min )}(Q)\left[e_{1}^{2}(t)+e_{2}^{2}(t)+e_{3}^{2}(t)\right] \\
& \leq-q_{(\min )}(Q) \sum_{1}^{3}\left|e_{i}(t)\right|^{2} .
\end{aligned}
$$

Using inequalities (49) and (50) that yields:

$$
\dot{V}(\boldsymbol{e}(t)) \leq-2 \frac{q_{(\min )}(Q)}{p_{(\max )}(P)} V(\boldsymbol{e}(t)) .
$$

Integrating the inequality (51) from $0 \rightarrow t$, results in the following Lyapunov estimation: 
where

$$
V(\boldsymbol{e}(t)) \leq V(0) \exp (-\Psi t)
$$

$$
\Psi=2 \frac{q_{(\min )}(Q)}{p_{(\max )}(P)} .
$$

Thus, the closed-loop system (41) is globally exponentially stable. This completes the proof of Theorem 5.1.

\subsection{Numerical simulation and discussion}

The parameters for the chaotic BVP oscillator [19] are set as $A=1, B=1.2$ and $\delta=1$ with initial states being taken as $x_{1}(t)=0.5, y_{1}(t)=1, z_{1}(t)=1.5$. Similarly, for the Chen chaotic oscillator [19], the parameters are set as $\alpha=35, \beta=3$, $\gamma=28$ and $v=7$, with initial states being taken as $x_{2}(t)=10$, $y_{2}(t)=-5, z_{2}(t)=38$. The gain of the controller is chosen as $k_{2}=26$. In numerical simulation, the following unknown external disturbances are applied to the master and slave systems (36), respectively.

$$
\begin{gathered}
D_{1}(t)=-0.2 \sin \left(\frac{3 \pi}{2} t\right), D_{2}(t)=-0.2 \sin (\pi t), \\
D_{3}(t)=0.2 \cos (\pi t), d_{1}(t)=0.15 \cos \left(\frac{\pi}{2} t\right), \\
d_{2}(t)=-0.1 \cos \left(\frac{2 \pi}{3} t\right), \quad d_{3}(t)=-0.2 \sin \left(\frac{\pi}{3} t\right) . \\
\text { Subsequently, } \Delta_{1}=0.35, \Delta_{2}=0.3 \text { and } \Delta_{3}=0.3 .
\end{gathered}
$$

Time history of the convergence of the error signals for non-identical synchronization (36) is illustrated in Figure 6. It can be noticed that the error states are synchronized with damped oscillation when the controllers are activated at $t=0.3 \mathrm{~s}$, app. This demonstrates that the investigated controllers are robust to accidental mismatches in the transmitter and receiver, which is helpful in secure communications. By numerical calculations, we can easily verify that; and $p_{(\max )}(P)=2.5$ and $q_{(\min )}(Q)=3$. Thus, from the main Theorem 3, we conclude that the two chaotic oscillators (36) are globally exponentially synchronized with an exponential convergence rate $\Psi=1.2$.

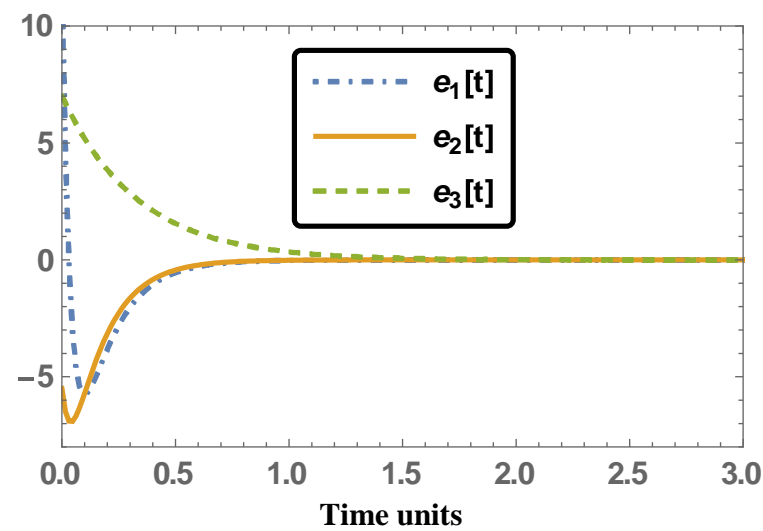

Fig. 6: Time series of the synchronized error states (BVP \& Chen systems)

\section{Conclusion}

This paper addressed the globally exponential synchronization of a doubled-scroll chaotic attractor exhibited by the three dimensional extended Bonhoeffer-van der Pol oscillator. Based on the Lyapunov direct method and using the active control technique, sufficient algebraic conditions were derived analytically to achieve the globally exponential synchronization. The globally exponential synchronization stability was verified theoretically and graphically. The capability of the synchronization stability is improved as compared to some previous results. It has been shown that the proposed active controller technique has strong robustness to the external disturbances and parameter mismatches.

Since chaotic signals are naturally broadband and noise-like, the synchronized chaotic oscillators can be used as encryption generators for secure communications. The results of this research work should be beneficial and could be employed in the field of hybrid image encryption, secure communications and genetic networks, etc.

The linear feedback gains are selected on trial-and-error bases. There are no suitable bounds for the linear feedback gains. Selecting of high linear feedback gains may create signal saturation in the systems. Similarly, there is no precise balance between the converging rates of the synchronized error signals and the magnitude of the linear feedback gains. These are open problems and should be addressed.

\section{References}

[1] Pecora L, Carroll T (1991), Synchronization in chaotic systems. $\begin{array}{llll}\text { Physics Review } & \text { Letters 64, }\end{array}$ https://doi.org/10.1103/PhysRevLett.64.821.

[2] Boccalettia S, Kurths J, Osipov G, Valladares DL, Zhou CS (2002), The synchronization of chaotic systems. Physics Reports 366, 201101. https://doi.org/10.1016/s0370-1573(02)00137-0.

[3] Xu Y, He Z (2013), Synchronization of variable-order fractional financial system via active control. Central European Journal of Physics 11, 6, 824-835. https://doi.org/10.2478/s11534-013-0237-x.

[4] Ahmad I, Saaban A, Ibrahim A, Shahzad M, Naveed N (2016), The Synchronization of chaotic systems with different dimensions by a robust generalized active control. Optik-International Journal for Light and Electron Optics 127, 11, 4859-4871. https://doi.org/10.1016/j.ijleo.2015.12.134.

[5] Li X (2009), Generalized projective synchronization using nonlinear control method. International Journal of Nonlinear Science 8, 1, 7985 .

[6] Ahmad I, Saaban A, Ibrahim A, Shahzad M (2016), Global chaos synchronization of new chaotic system using linear active control. Complexity 21, 1, 379-386. https://doi.org/10.1002/cplx.21573.

[7] Ahmad I, Saaban A, Ibrahim A, Shahzad M, Alsawalha M (2016), Robust reduced-order synchronization of times-delay chaotic systems with known and unknown parameters, Optik-International Journal for Light and Electron Optics 127, 13, 5506-5514. https://doi.org/10.1016/j.ijleo.2016.02.078.

[8] Yan, ZY (2005), a new scheme to generalize (lag, anticipated, and complete) synchronization in chaotic and hyperchaotic systems. Chaos 15:013101-013110. https://doi.org/10.1063/1.1827431.

[9] Zhang B, Li H (2014), Universal function projective synchronization of two different hyperchaotic systems with unknown parameters. Journal of Applied Mathematics, Article ID 549201, 10 pages.

[10] Chen Y, Li M, Cheng Z (2010), Global anti-synchronization of master-slave chaotic modified Chua's circuits coupled by linear feedback control. Mathematical and Computer Modeling 52, 3-4, 567573. https://doi.org/10.1016/j.mcm.2010.03.056.

[11] Ho M, Hung Y (2001), Synchronization of two different systems by using generalized active control. Physics Letters A 301, 424-428. https://doi.org/10.1016/S0375-9601(02)00987-8.

[12] Chen H (2002), Chaos and chaos synchronization of a symmetric gyro with linear-plus-cubic damping. Journal of Sound and Vibration 255, 719-740. https://doi.org/10.1006/jsvi.2001.4186.

[13] Ucar A, Lonngren KE, Bai EW (2007), Chaos synchronization in RCL-shunted Josephson junction via active control. Chaos, Solitons and Fractals 31, 105-111. https://doi.org/10.1016/j.chaos.2005.09.035

[14] Vincent UE, Ucar A (2007), Synchronization and anti-synchronization of chaos in permanent magnet reluctance machine. Far East Journal of Dynamical Systems 9, 211-221.

[15] Njah AN, Vincent UE (2009), Synchronization and anti-synchronization of chaos in an extended Bonhoffer-van der Pol oscillator using active control. Journal of Sound and Vibration 319, 493-504. https://doi.org/10.1016/j.jsv.2008.05.036.

[16] Khalil HK (2002), Nonlinear Systems, 3rded. Prentice Hall, New Jersey.

[17] Anderson P, Fouad A (1984), Power System Control and Stability, Iowa State University Press, Ames, Iowa.

[18] Liao X, Yu P (2006), Study of globally exponential synchronization for the family of Rossler systems. International Journal of Bifurcation and Chaos 16, 8, 2395-2406. https://doi.org/10.1142/s0218127406016148.

[19] Zhou T, Chen G (2006), Classification of chaos in 3-D autonomous quadratic system-1. Basic framework and method. International Journal of Bifurcation and Chaos 16(9) 2456-2479, 2006. https://doi.org/10.1142/S0218127406016203. 
[20] Wang Q, You Y, Wang H (2014), robust synchronization of hyperchaotic systems with uncertainties and external disturbances. Journal of Applied Mathematics. Article ID 523572, 8 pages.

[21] Boyd S (2004). Convex optimization, Cambridge University Press, NY, USA. https://doi.org/10.1017/CBO9780511804441.

[22] Wirkus S, Rand R (2002), the dynamics of two coupled Van der Pol oscillators with delay coupling. Nonlinear Dynamics 30, 205-221. https://doi.org/10.1023/A:1020536525009.

[23] Sekikawa M, Inaba N, Yoshinaga T, Hikihara T (2010), Period-doubling cascades of canards from the extended Bonhoeffer-van der Pol oscillator. Physics Letters A 374, 36, 3745-3751. https://doi.org/10.1016/j.physleta.2010.07.033.

[24] Sekikawa M, Shimizu K, Inaba N, Kita H, Endo T, Fujimoto K Yoshinaga T, Aihara K (2011), Sudden change from chaos to oscillation death in the Bonhoeffer-van der Pol oscillator under weak periodic perturbation. Physics Review E 84, 056209. https://doi.org/10.1103/physreve.84.056209.

[25] Ramesh M, Narayanan S (2001), Chaos control of Bonho“ ffer-van der Pol oscillator using neural networks. Chaos, Solitons and Fractals 12, 2395-2405. https://doi.org/10.1016/S0960-0779(00)002009. 